\title{
Antiviral Activity and Mechanism of Action of Endoplasmic Reticulum Glucosidase Inhibitors: A Mini Review
}

\author{
Momoko Onda; and Wataru Hakamata \\ Department of Chemistry and Life Science, College of Bioresource Sciences, Nihon University, \\ Fujisawa-shi, Kanagawa 252-0880, Japan \\ FAX: +81-466-84-3960, E-mail: hakamata.wataru@nihon-u.ac.jp
}

(Received on May 25, 2018, accepted on June 25, 2018)

Key Words: antiviral activity, endoplasmic reticulum glucosidase, inhibitor, direct-acting antivirals, host-targeting antivirals

\begin{abstract}
Antivirals are used to treat viral infections, and antibiotics are used to treat bacterial infections. However, the mechanisms of action and number of commercially available antivirals are very limited compared to those for antibiotics. Accordingly, our group is engaged in ongoing research to develop host-targeting antivirals for the virus infections. This work is primarily focused on the endoplasmic reticulum (ER) glucosidases involved in $N$-glycan synthesis as the host-dependent factors of viral infection. It is widely accepted that a key mechanism by which those inhibitors act as antivirals is their ability to disrupt virus glycoprotein folding via their inhibition of ER glucosidases. Importantly, very few virus strains are resistant to ER glucosidase inhibitors because ER glucosidase enzymes are not encoded on virus genomes. This avoids problems arising from resistance mutations occurring in the viral target. A number of ER glucosidase inhibitors with antiviral activity have been reported in the past, and several clinical trials of these have been performed. In this paper, we examine the factors preventing the development of these inhibitors as antivirals and our attempts to overcome them.
\end{abstract}

\section{A. Introduction}

In the latter half of the 1970 s, it became recognized that the global spread of newly emerging viral infections, such as highly pathogenic influenza, severe acute respiratory syndrome, Middle East respiratory syndrome, and Ebola hemorrhagic fever, as well as re-emerging viral infections such as West Nile fever, dengue fever, and Zika fever, was becoming increasingly difficult to control (1). Furthermore, viruses that cause infection include not only emerging and re-emerging viruses but also influenza viruses, hepatitis B and $\mathrm{C}$ viruses, herpes, and human immunodeficiency virus (HIV), among others.

Anti-influenza and anti-HIV drugs, are relatively well researched. However, the number of antivirals for other virus infections including emerging and re-emerging virus infections is currently very limited. This is largely due to the difficulties associated with the development of antivirals. These difficulties include 1) the simple structures of virus, which present few antivirals targets; 2) the diversity of viruses, the various genomic forms of which have different life cycles; 3) host-dependent viral proliferation, i.e., the host cell biological processes being hijacked by the virus; 4) the fast mutation rate of viral genomes (for example, the gene of HIV1 mutates at a rate over 1,000,000 times that of eukaryotic organisms and DNA virus genes) (2); 5) the diversity of virus infections and their proliferation processes, which makes it very difficult to develop antivirals with wide antiviral spectra; and 6) the limited market of antivirals (even for viral infections with a large number of sufferers, the pharmaceutical market is relatively small). These reasons also lead to a limited number of antivirals studies being published in academic journals (3).

In this paper, we discuss the use of endoplasmic reticulum (ER) glucosidase inhibitors as antivirals and the mechanism of their antiviral activity. In addition, we review our efforts to develop antivirals through screening and structural development of ER glucosidase inhibitors.

\section{B. Host-Targeting Antivirals}

Direct-acting antivirals (DAAs), which directly target viral proteins, are being continuously developed (4). The major DAAs can be categorized depending on their mode of action as protease inhibitors, nucleic acid synthesis inhibitors (i.e., DNA and RNA synthetase, and reverse transcriptase inhibitors), or non-structural protein inhibitors (i.e., inhibitors of proteins involved in the synthesis of viral RNAs and inhibitors of viral protein associations, as opposed to enzyme inhibitor). DAAs exhibit selective toxicity toward the virus because they target the virus proteins. However, this means that they inevitably suffer from issues due to resistance mutations arising in the viral target. Viral polymorphism can appear naturally in regions targeted by DAAs, conferring DAA resistance. Multi-drug combination therapy is viable response to the emergence of such drug-resistant strains.

Host-targeting antivirals (HTAs), which target proteins involved in virus infection and proliferation in human host cells, are attractive options for addressing the appearance of drug-resistant viral strains (5). HTAs provide a broad antiviral activity with very 


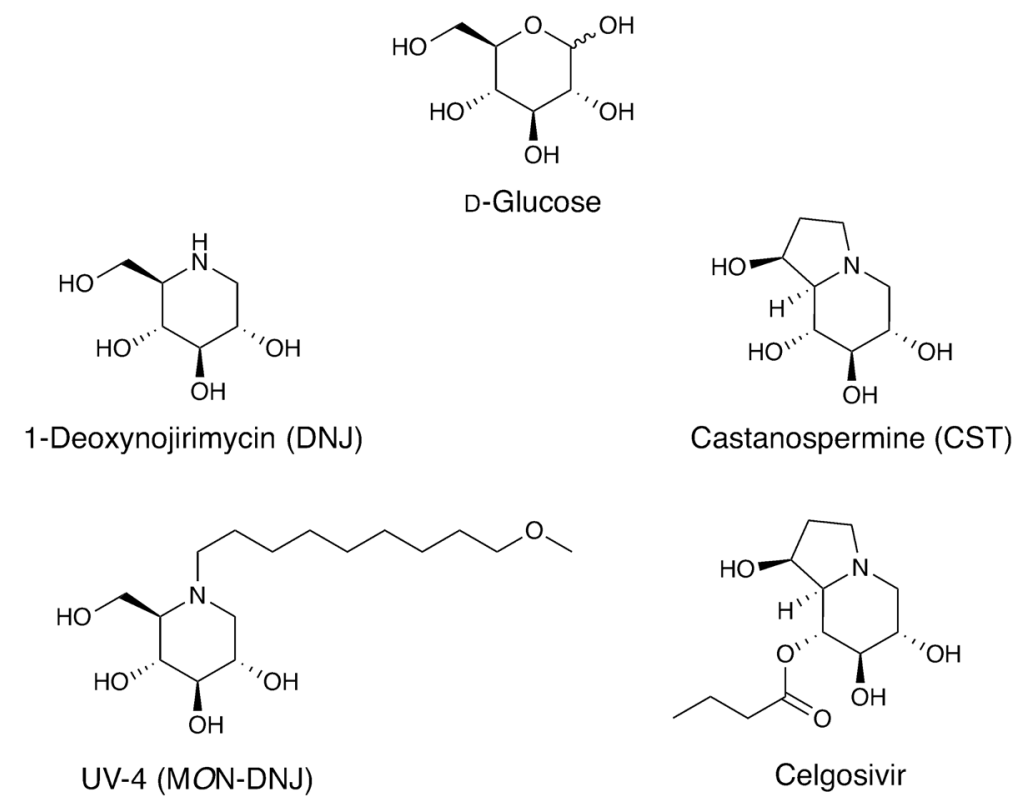

Fig. 1. Structures of D-glucose and ER GI and ER GII inhibitors.

high genetic barriers to drug resistance owing to the extremely low mutational rate of host cells (6). However, the development of HTAs that exhibit selective toxicity toward the virus is challenging.

\section{Inhibitors of $\mathrm{N}$-Glycan Processing Enzymes as HTAs}

$N$-glycan processing is performed by hydrolytic enzymes and transferases in the ER and Golgi apparatus in human cells. These processes are potential targets for HTAs. Their inhibition can disrupt the assembly and/or release steps in the virus life cycle, leading to antiviral activity.

The proliferation of "enveloped" viruses, which constitute approximately $70 \%$ of viruses isolated from animals, is particularly well inhibited by this mechanism of action (7). Virus envelopes consist of lipids and glycoproteins from the host cell membrane, and virus-derived glycoproteins. $\mathrm{N}$-glycans on the glycoproteins in the envelopes play important roles in virus functions, such as hostcell-receptor binding and suppression of biological defense mechanisms by the host cells. 1-Deoxynojirimycin (DNJ) and casutanospermine (CST) were the first-reported anti-HIV agents that work based on ER glucosidase I (ER GI) and glucosidase II (ER GII) inhibition $(8,9)$. The ER GI and ER GII are involved in the early stages of $N$-glycan processing. Around the 1990s, many clinical trials of ER GI and ER GII inhibitors were undertaken. Recently, Phase II clinical trials for UV-4 (MON-DNJ), a derivative of DNJ, and Celgosivir, a derivative of CST, have been performed. The clinical trials of Celgosivir were stopped in the 2010 (10). These inhibitor structures are summarized in Fig. 1.

\section{Antiviral Mechanism of ER GII Inhibitors}

Normally, virus envelope glycoproteins undergo correct protein folding through ER protein quality control (ERQC) as a host cell mechanism. Subsequently, the correctly folded glycoproteins are transported to the Golgi apparatus and undergo post-translational modifications (PTMs). Thus, the antiviral action of ER GI and ER GII inhibitors is based on the disruption of viral glycoprotein folding. Consequently, ER GI and ER GII are very promising targets for the development of broad-spectrum antivirals for enveloped viruses.

Glycoprotein folding is crucial for virus assembly and release in the virus life cycle. This antiviral action is summarized in Fig. 2. Briefly, nascent proteins are modified at the lumen of the ER through the addition of glycans in the form of $\mathrm{Glc}_{3} \mathrm{Man}_{9} \mathrm{GlcNAc}_{2}$ by oligosaccharyltransferase. The $N$-glycan is first hydrolyzed at a glucose residue by ER GI to give $\mathrm{Glc}_{2} \mathrm{Man}_{9} \mathrm{GlcNAc}_{2}$ and then at another glucose residue by ER GII to give $\mathrm{Glc}_{1} \mathrm{Man}_{9} \mathrm{GlcNAc}_{2}$. This monoglucosylated glycan is recognized on the virus protein by the calnexin/calreticulin (CNX/CRT) quality control system, confirming correct protein folding and allowing transport to the Golgi apparatus.

When a virus proliferates in human cells, ER GII inhibition leads to more $\mathrm{Glc}_{2} \mathrm{Man}_{9} \mathrm{GlcNAc}_{2}$ on the viral glycoproteins than usual. This diglucosylated glycan can be specifically bound for malectin, a membrane-bound ER-resident lectin that is induced by the unfolded protein response based on ER stress (11). The induced 


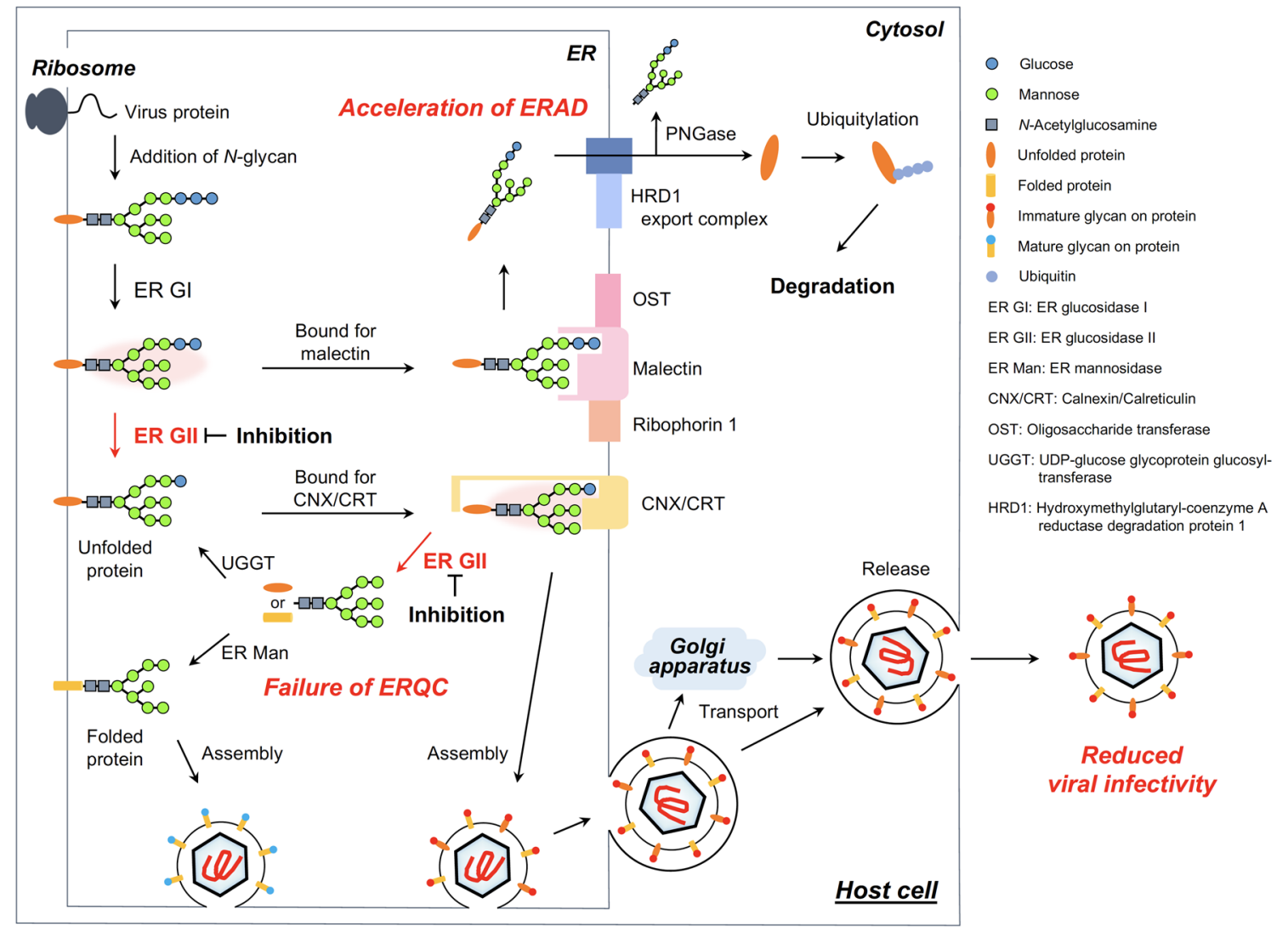

Fig. 2. Schematic illustrating the mechanism of antiviral action for ER GII inhibitors.

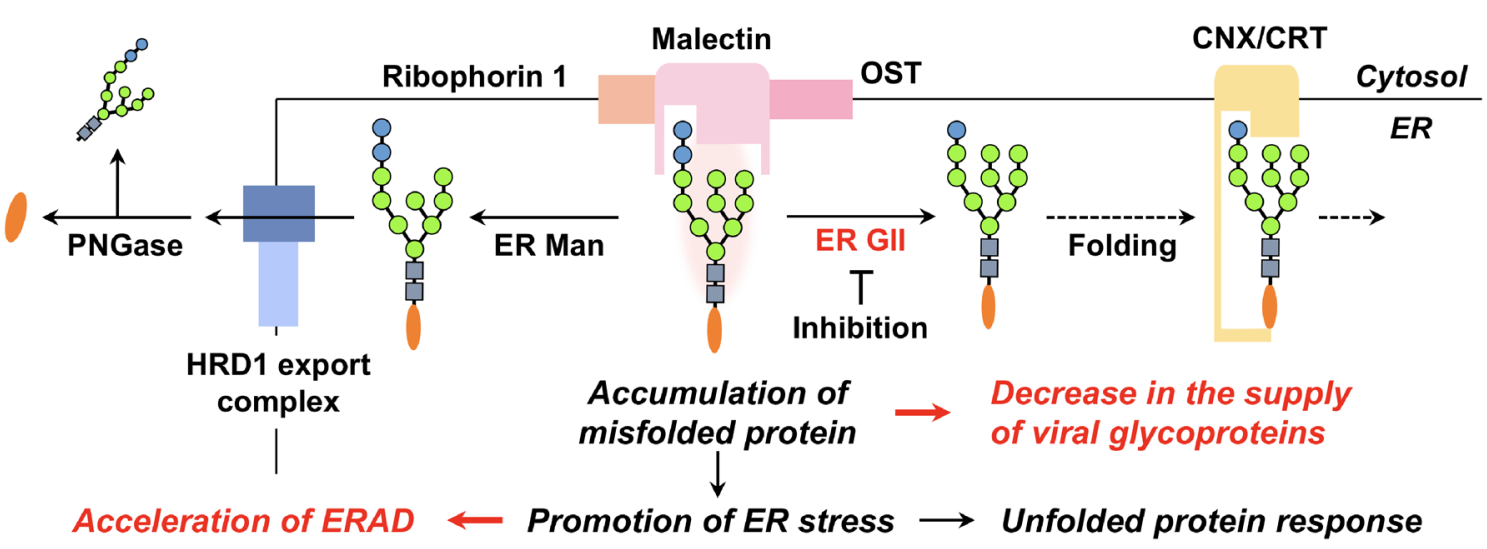

Fig. 3. Accelerated ERAD and decrease in the supply of viral glycoproteins by the accumulation of diglucosylated $N$-glycan on viral proteins.

malectin is thought to preferentially associate with misfolded proteins, retaining them in the ER (12). As a result, the appropriate supply of viral glycoproteins for the capsid and envelope is delayed by the accelerated ER-associated degradation (ERAD). The delay causes a decrease in the production of viral particles, which is sufficiently fatal to antiviral action. This concept is illustrated in Fig. 3.

Inhibition of ER GII reduces the quantity of
$\mathrm{Glc}_{1} \mathrm{Man}_{9} \mathrm{GlcNAc}_{2}$ in the ER, which is important for binding CNX/ CRT and correct protein folding, and the amount of correctly folded viral capsid and envelope glycoproteins in the ER. At the same time, the ER GII inhibitor also inhibits the ER GII involved in the $\mathrm{CNX} / \mathrm{CRT}$ cycle, and its function is greatly reduced. Consequently, the ERQC mechanism collapses. Therefore, the decreased in the supply of viral capsid and envelope glycoproteins in the ER prevents the assembly of viral particles. This concept is illustrated in 


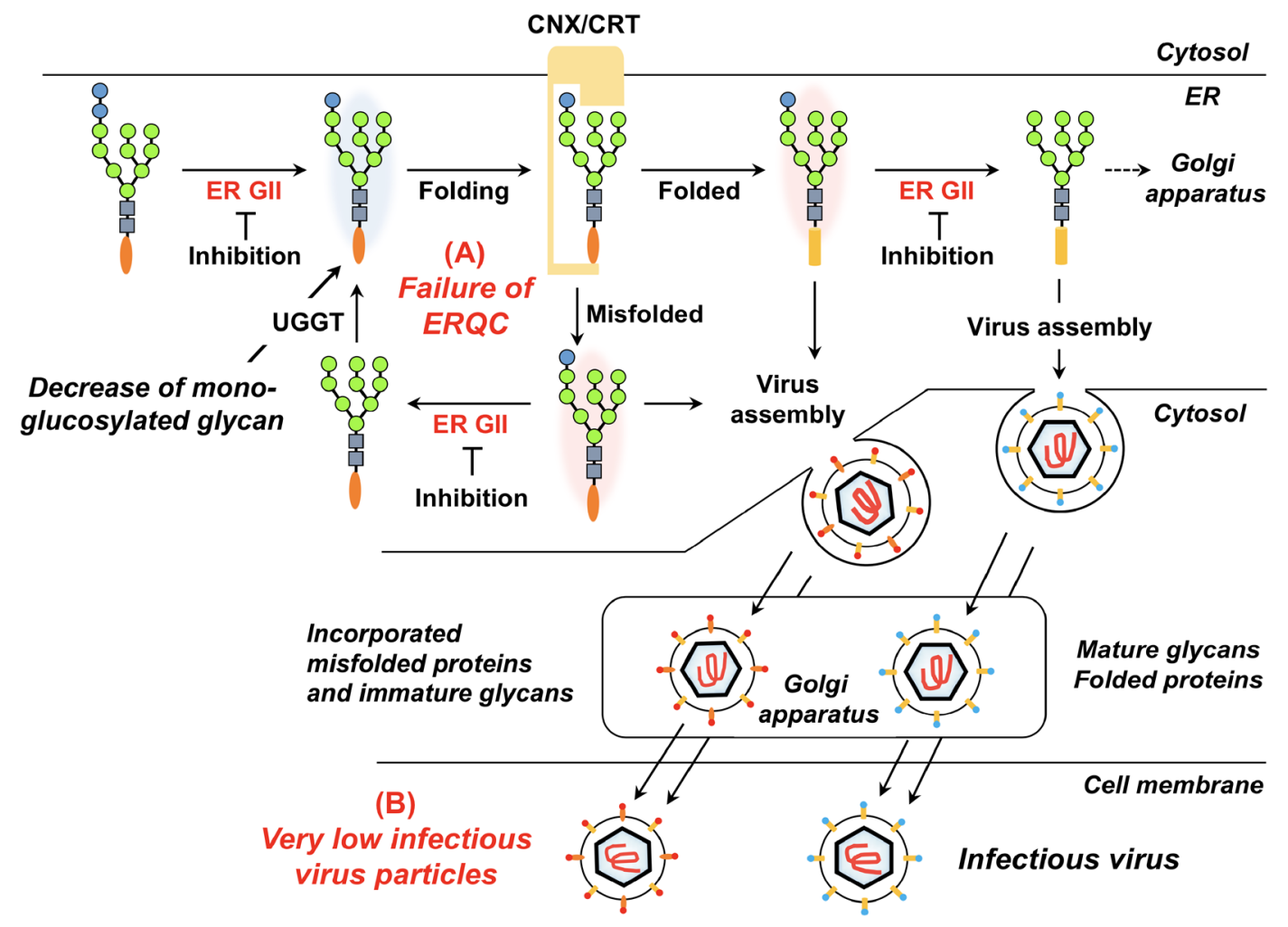

Fig. 4. (A) ERQC failure by ER GII inhibition, (B) secretion of very low infectious virus particles incorporating immature glycans.

Fig. 4 (A). It should be noted that, even under such circumstances, many virus species can assemble and release virus particles. However, viruses released from cells with immature glycans in their envelope proteins exhibit greatly reduced infectivity toward other cells. This concept is illustrated in Fig. 4 (B).

However, there are details of the antiviral mechanism of ER GII inhibition that are still not fully understood. For example, the $\mathrm{Glc}_{2} \mathrm{Man}_{9} \mathrm{GlcNAc}_{2}$ that accumulates under ER GII inhibition can be hydrolyzed to $\mathrm{Man}_{8} \mathrm{GlcNAc}_{2}$ by endomannosidase in cells. Thus, the viral glycoproteins bearing $\mathrm{Man}_{8} \mathrm{GlcNAc}_{2}$ can be folded correctly and receive normal PTMs at the Golgi apparatus. Furthermore, viruses with immature glycans can be released without passing through PTMs at the Golgi apparatus. In addition, the contributions of the three different mechanisms of action (Figs. 3 and 4) to antiviral activity are far from clear and are thought to depend on the kind of virus and inhibitor. Thus, antiviral activity by ER GII inhibition has not yet been sufficiently elucidated and requires further research effort.

\section{E. Enzymatic Differences between ER GI and ER GII}

The enzymatic properties of ER GI and ER GII differ greatly. An ER GI (EC 3.2.1.106) is an inverting glycoside hydrolase classified into glycoside hydrolase family 63 (GH 63). An ER GII (EC 3.2.1.84) is a retaining glycoside hydrolase classified into GH 31.
Furthermore, these enzymes differ in their substrate specificity. ER GI hydrolyzes only oligosaccharides, whereas ER GII hydrolyzes both oligosaccharides and monosaccharide derivatives (13). Generally, a fluorescent substrate is used to measure the activities of these enzymes for the purpose of designing, screening, and evaluating inhibitors. The development of substrates for ER GI that produce fluorescence upon enzyme hydrolysis requires the incorporation of a means of fluorescence resonance energy transfer in the substrate structure, which is highly difficult in terms of both design and synthesis (14). However, a fluorescent substrate for ER GII need only be a monosaccharide derivative that releases a fluorophore upon enzyme hydrolysis, and this is much easier to achieve. Furthermore, the relative contributions to antiviral activity made by the inhibition of ER GI and ER GII are still not clear (15).

\section{F. Molecular Features and Intracellular Targets of ER GII Inhibitor}

Many ER GII inhibitors have undergone clinical trials, but none have reached Phase III. The main reason for this problem of clinical trials is the low uptake of the inhibitors into the ER of human cells (15). For human host cells, an ER GII inhibitor must penetrate two membranes, the cell membrane and the ER membrane, to reach target enzymes in the ER. Hydrophobicity is generally required for membrane permeation. Accordingly, MON-DNJ 


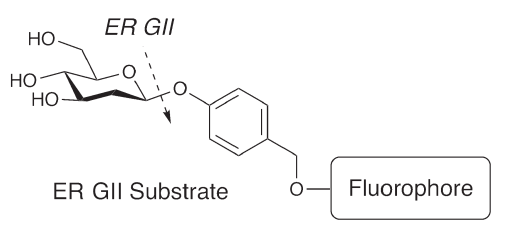

Fluorescence off
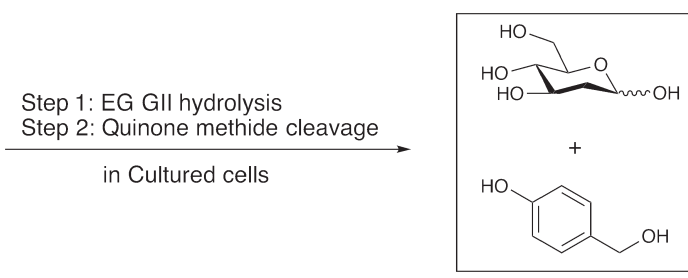

Fluorophore

Strong fluorescence

in cultured cells

Fig. 5. Quinone methide cleavage platform for ER GII substrate.

is modified version of DNJ that bears an alkyl-chain to enhance its hydrophobicity. Furthermore, Celgosivir is essentially an alkylchain ester of CST with enhanced hydrophobicity that acts as a prodrug.

Naturally, inhibitors that mimic glucose must have essentially glucose-like structures, and this presents a limit for potency improvement based on structural development. Numerous studies on the structural development of such inhibitors have been reported (16). However, the structural diversity of glucose-mimicking inhibitors is intrinsically bound by this limit. Thus, non-substratemimicking inhibitors are attracting increasing attention as nextgeneration inhibitors owing to their design flexibility. This flexibility makes it possible to impart hydrophobicity to an inhibitor and to decrease its number of asymmetric centers (17).

ER GII inhibitors with or without substrate-mimicking structures must have the ability to bind glucose recognition proteins. Thus, because of the extensive use of glucose in cells, the likelihood of such inhibitors having multiple binding partners is high. Accordingly, ER GII inhibitors have been reported to have binding affinity with other glucose recognition enzymes, including maltase, isomaltase, sucrase, and cellobiase in the small intestine, lysosomal $\alpha$-glucosidase, ceramide glucosyltransferase, and glucocerebrosidase as off-targets $(15,18)$. Among them, the inhibition of glycolipid processing is not necessarily defined as off-target as glycolipids play an important role in the life cycle of many viruses. It is therefore possible that the antiviral effects of ER GII inhibitors arise from the inhibition of glycolipid processing in addition to inhibiting glycoprotein folding (19). However, anti-dengue virus activity has been reported to require inhibition of glycan processing alone and not inhibition of glycolipid processing (20).

\section{G. Screening Strategy for ER GII Inhibitors}

A promising strategy for obtaining non-substrate-mimicking ER GII inhibitors is virtual ligand screening using the X-ray crystal structure of the catalytic unit of ER GII as a template (21). Another important strategy is the screening of compound libraries, which requires a system for evaluating inhibitory activity and, naturally, a compound library. In Japan, the Drug Discovery Initiative of the University of Tokyo and Drug Discovery Chemical Bank Unit of the Institute of Physical and Chemical Research (RIKEN) provide compound libraries for researchers. In vitro assay systems using pure isolated ER GII are suitable for high-throughput (HT) screening of ER GII inhibitors. However, there are no reports on the isolation of the ER GII with glucosidase activity from human cells.

Generally, measurement of ER GII activity involves the use of the suitable monosaccharide derivative as an ER GII substrate and the microsomal fraction of rat liver as an enzyme source (13). However, HT inhibitor screening of a compound library using crude enzymes that rapidly deactivate is difficult. We have focused on human cultured cells as a source of the ER GII enzyme for HT ER GII inhibitor screening. Consequently, the compounds identified by such HT inhibitor screening show promise as inhibitors of human ER GII and exhibit excellent membrane permeability. However, there are no reports of fluorescent ER GII substrates that work with human cultured cells. Therefore, we have performed structural analysis on the reported X-ray crystal structure of the active site in ER GII catalytic unit $(22,23)$ and used these data in an attempt to design a fluorescent substrate for ER GII. Consequently, we proposed a quinone methide cleavage (QMC) platform, which is a molecular design platform for ER GII substrates suitable for ER GII active sites involved in subsite -1 and subsite +1 (2426). This concept is illustrated in Fig. 5. Moreover, we have investigated the substrate specificity of ER GII and found that it also exhibits 2-deoxyglucosidase activity (13). Thus, we designed and synthesized a fluorescent substrate for ER GII to work with human cultured cells, taking advantage of the 2-deoxyglucosidase activity and the QMC platform. We then screened ER GII inhibitors from compound libraries using the fluorescent substrate. As a result, a non-substrate-mimicking inhibitor showing ER GII inhibitory activity in human cultured cells was discovered.

\section{H. Closing Remarks}

Antivirals are desperately required to treat viral infections, including newly emerging and re-emerging viral infections as well as 
currently unknown viral infections and those potentially exploited in viral bioterrorism. Currently, DAAs are used very effectively for these viral infections. In the future, HTAs will become an important antiviral option for use alongside DAAs. Our group is committed to developing and providing ER GII inhibitors as HTAs.

\section{Acknowledgments}

This study is supported by JSPS KAKENHI Grant Number 26460157 and 17K08375, and Nihon University College of Bioresource Sciences Research Grant for 2017.

\section{References}

1. Rezza, G., and Ippolito, G. (Eds.). (2017) Emerging and Re-emerging Viral Infections in Advances in Microbiology, Infectious Diseases and Public Health, vol. 6, Springer.

2. Korber, B., Muldoon, M., Theiler, J., Gao, F., Gupta, R., Lapedes, A., Hahn, B. H., Wolinsky, S., and Bhattacharya, T. (2000) Science 288, 17891796.

3. Watashi, K. (2013) Yakugaku Zasshi 113, 1169-1175.

4. Martinez, J. P., Sasse, F., Brönstrup, M., Diez, J., and Meyerhans, A. (2015) Nat. Prod. Rep. 32, $29-48$.

5. Ahmed, A., and Felmlee, D. J. (2015) Viruses 7, 6716-6729.

6. Zeisel, M. B., Crouchet, E., Baumert, T. F., and Schuster, C. (2015) Viruses 7, 5659-5685.

7. Granoff, A., and Webster, R. G. (Eds.). (1994) Encyclopedia of virology, Academic Press Inc.

8. Gruters, R. A., Neefjes, J. J., Tersmette, M., De Goede, R. E. Y., Tulp, A., Huisman, H. G., Miedema, F., and Ploegh, H. L. (1987) Nature 330, 74-77.

9. Walker, B. D., Kowalski, M., Goh, W. C., Kozarsky, K., Krieger, M., Rosen, C., Rohrschneider, L., Haseltine, W. A., and Sodroski, I. (1987) Proc. Natl. Acad. Sci. U.S.A. 84, 8120-8124.

10. Crouchet, E., Wrensch, F., Schuster, C., Zeisel, M. B., and Baumert, T. F. (2018) Therap. Adv. Gastroenterol. 11, 1756284818759483.

11. Schallus, T., Jaeckh, C., Fehér, K., Palma, A. S., Liu, Y., Simpson, J. C., Mackeen, M., Stier, G., Gibson, T. J., Feizi, T., Pieler, T., and Muhle-Goll, C. (2008) Mol. Biol. Cell 19, 3404-3414.

12. Słomińska-Wojewódzka, M., and Sandvig, K. (2015) Molecules 20, 9816-9846.

13. Hakamata, W., Muroi, M., Nishio, T., Oku, T., and Takatsuki, A. (2004) J. Carbohydr. Chem. 23, 27-39.

14. Yang, G. Y., Li, C., Fischer, M., Cairo, C. W., Feng, Y., and Withers, S. G. (2015) Angew. Chem. Int. Ed. Engl. 54, 5389-5393.

15. Alonzi, D. S., Scott, K. A., Dwek, R. A., and Zitzmann, N. (2017) Biochem. Soc. Trans. 45, 571-582.

16. Horne, G., Wilson, F. X., Tinsley, J., Williams, D. H., and Storer, R. (2011) Drug Discov. Today 16, $107-118$.

17. Koyama, R., and Hakamata, W. (2017) SAIBOU 49, 720-724.

18. Kiappes, J. L., Hill, M. L., Alonzi, D. S., Miller, J. L., Iwaki, R., Sayce, A. C., Caputo, A. T., Kato, A., and Zitzmann, N. (2018) ACS Chem. Biol. 13, 60-65.

19. Mellor, H. R., Neville, D. C., Harvey, D. J., Platt, F. M., Dwek, R. A., and Butters, T. D. (2004) Biochem. J. 381, 867-875.

20. Sayce, A. C., Alonzi, D. S., Killingbeck, S. S., Tyrrell, B. E., Hill, M. L., Caputo, A. T., Iwaki, R., Kinami, K., Ide, D., Kiappes, J. L., Beatty, P. R., Kato, A., Harris, E., Dwek, R. A., Miller, J. L., and Zitzmann, N. (2016) PLoS Negl. Trop. Dis. 10, e0004524.

21. Hakamata, W., Ishikawa, R., Ushijima, Y., Tsukagoshi, T., Tamura, S., Hirano, T., and Nishio, T. (2012) Bioorg. Med. Chem. Lett. 22, 62-64.

22. Satoh, T., Toshimori, T., Noda, M., Uchiyama, S., and Kato, K. (2016) Protein Sci. 25, $2095-2101$.

23. Caputo, A. T., Alonzi, D. S., Marti, L., Reca, I. B., Kiappes, J. L., Struwe, W. B., Cross, A., Basu, S., Lowe, E. D., Darlot, B., Santino, A., Roversi, P., and Zitzmann, N. (2016) Proc. Natl. Acad. Sci. U.S.A. 113, E4630-E4638.

24. Hakamata, W., Tamura, S., Hirano, T., and Nishio, T. (2014) ACS Med. Chem. Lett. 5, 321-325.

25. Hakamata, W., Miura, K., Hirano, T., and Nishio, T. (2015) Bioorg. Med. Chem. 23, 73-79.

26. Miura, K., Hakamata, W., Tanaka, A., Hirano, T., and Nishio, T. (2016) Bioorg. Med. Chem. 24, 1369-1375. 


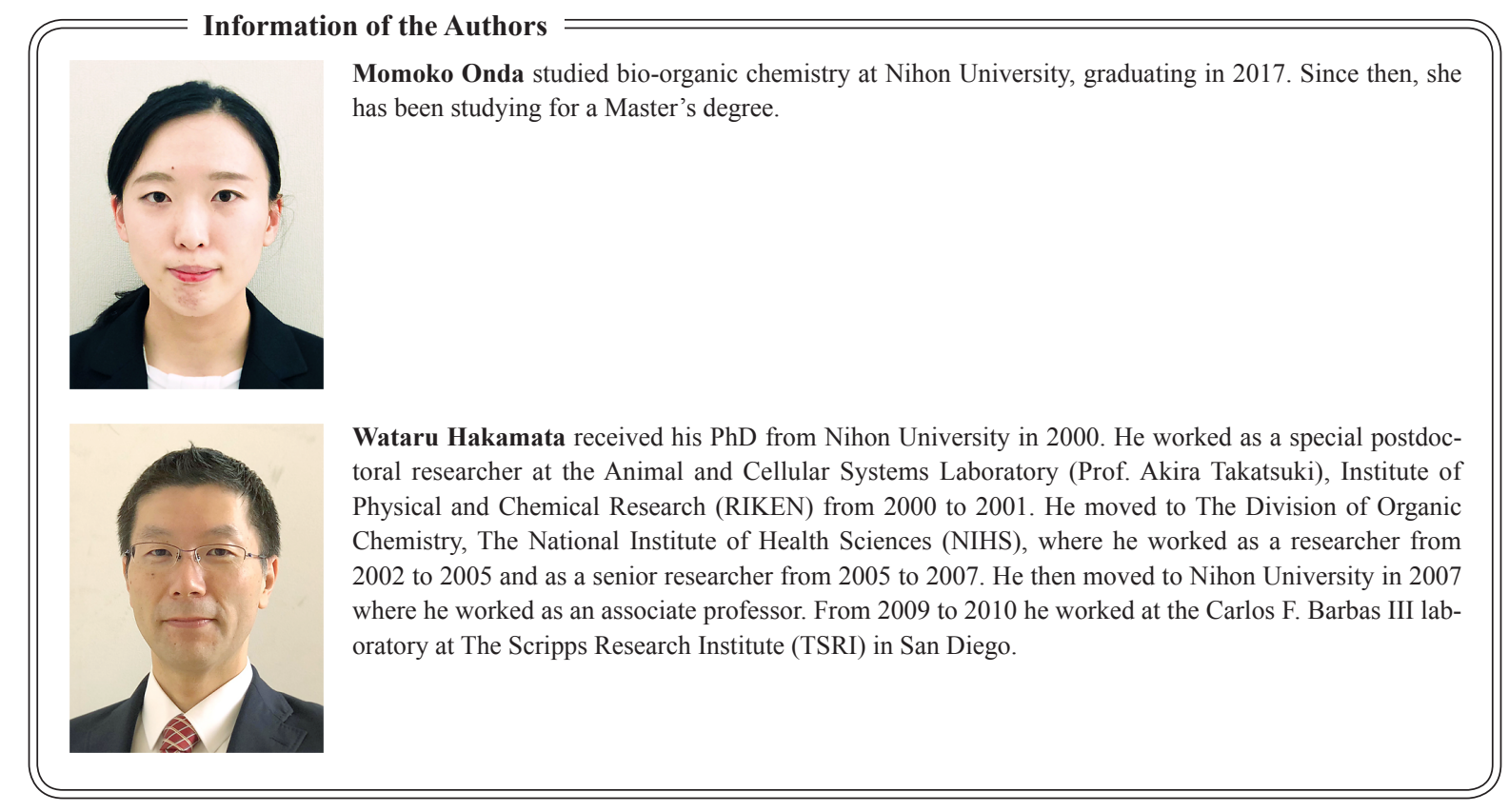

